
du F.L.E. (18e et 19 e siècles)

\title{
À l'origine de l'histoire du FLE en Espagne. A propos de G. Suárez Gómez
}

Juan Francisco García Bascuñana, Brigitte Lépinette et Carmen Roig

\section{(2) OpenEdition}

Journals

Édition électronique

URL : https://journals.openedition.org/dhfles/3008

DOI : $10.4000 /$ dhfles.3008

ISSN : 2221-4038

Éditeur

Société Internationale pour l'Histoire du Français Langue Étrangère ou Seconde

Édition imprimée

Date de publication : 1 décembre 1999

Pagination : p. 59-73

ISSN : 0992-7654

Référence électronique

Juan Francisco García Bascuñana, Brigitte Lépinette et Carmen Roig, « À l'origine de l'histoire du FLE en Espagne. A propos de G. Suárez Gómez », Documents pour l'histoire du français langue étrangère ou seconde [En ligne], 24 | 1999, mis en ligne le 17 janvier 2015, consulté le 27 mai 2021. URL : http:// journals.openedition.org/dhfles/3008 ; DOI : https://doi.org/10.4000/dhfles.3008

Ce document a été généré automatiquement le 27 mai 2021.

(c) SIHFLES 


\title{
À l'origine de l'histoire du FLE en Espagne. A propos de G. Suárez Gómez
}

\author{
Juan Francisco García Bascuñana, Brigitte Lépinette et Carmen Roig
}

1 L'histoire de la présence du français en Espagne et, tout particulièrement, celle de son enseignement ont été, jusqu'à ces dernières années relativement peu étudiées en Espagne. Cependant, si l'histoire du français langue étrangère (dorénavant FLE) représente de ce côté des Pyrénées un domaine de recherche encore assez nouveau, il a connu récemment un essor considérable dans d'autres pays européens. De ce point de vue, la fondation de la SIHFLES sous les auspices d'André Reboullet et Daniel Coste entre autres- en 1988, a sans aucun doute constitué un événement capital qui a permis, d'abord, d'intégrer dans un cadre de recherche commun de nombreux travaux auparavant dispersés dans des publications diverses (à l'étranger ou en France), ensuite de susciter de nouvelles investigations. C'est en Allemagne et en Italie où parurent les premiers travaux qui traitèrent, dans une perspective historique, la question de la présence et de l'enseignement du français dans leurs pays respectifs. Nous voudrions, tout d'abord, mentionner les chercheurs allemands, spécialement Herbert Christ, président de la SIHFLES entre 1991 et 1993, dont les apports à l'histoire du FLE nous semblent fondamentaux. La vaste bibliographie ${ }^{1}$ que signe ce grand historien $\mathrm{du}$ français dans les pays germaniques témoigne de l'ampleur de la recherche effectuée. Nous rappellerons tout particulièrement ses travaux sur la méthode directe et l'influence décisive qu'ils ont eue dans l'enseignement des langues étrangères, et plus précisément du FLE. Outre ces précieuses contributions de H. Christ, l'histoire du FLE en Allemagne a aussi été abordée par différents groupes de recherche qui ont également àleur actif des travaux hautement intéressants.

2 Tout autre est le cas de la Suède pour lequel nous nous trouvons, non face à un travail collectif, mais à celui -solitaire- d'Elisabet Hammar qui a su compenser son isolement par un effort digne de tout éloge: son livre, L'enseignement du français en Suède jusqu'en 
1807. Méthodes et manuels -connu de tous- donne la mesure de l'intérêt et de l'importance de son investigation ${ }^{2}$.

3 L'Italie a vu surgir une équipe de recherche particulièrement imposante. En réalité, il s'agit plutôt d'un réseau que d'une équipe unique et unifiée. Ce réseau est constitué par divers groupes qui gravitent autour de Bologne, et dont les noms les plus souvent cités sont ceux de Nadia Minerva et de Carla Pellandra. La valeur de ces recherches se manifeste dans les multiples études publiées par ces collègues italiennes -eh! oui, toutes sont des femmes ..qui ont pour objet surtout les $18^{\mathrm{e}}$ et le $19^{\mathrm{e}}$ siècles $^{3}$, mais qui, parfois, vont également au-delà dans le temps. Les travaux sur la période napoléonienne, en particulier, nous semblent un modèle d'approche méthodologique. Ils sont aussi spécialement intéressants du point de vue des résultats.

4 L'Espagne est loin d'être absente dans cet effort collectif récent pour explorer l'histoire de l'enseignement du français dans la Péninsule Ibérique. En attendant des réflexions plus complètes et plus spécifiques concernant notre espace géographique ${ }^{4}$, nous tenterons ici de décrire brièvement certains des apports espagnols à l'histoire du FLE. En dépit de ce qu'on pourrait peut-être croire, l'intérêt pour ce domaine de recherche n'est pas récent chez nous. Avant même 1988, date emblématique pour la SIHFLES, il existe des travaux sur l'histoire du FLE. Certes, on n'y trouve pas, jusqu'à ces toutes dernières années, de groupes de recherche organisés contrairement à ce que nous avons pu constater pour l'Allemagne et spécialement pour l'Italie, mais des tentatives, plus ou moins isolées (et il faut le dire, plus ou moins heureuses) ont vu le jour depuis une quinzaine d'années, avant que la SIHFLES n'ait pu les intégrer dans son cadre à la fois protecteur et stimulant. Mais au lieu d'avancer ici des noms, ou de mentionner des démarches pionnières qui ont surgi, éparses, à partir de la seconde moitié des années 1980, acquérant une vigueur indéniable, nous devons citer un apport fondamental, qui peut même être considéré fondateur. C'est la thèse de doctorat de Gonzalo Suárez Gómez (G.S.G. dorénavant) dont parlait précisément le texte d'André Reboullet qui aété lu lors de la séance d'ouverture de ce colloque.

La thèse de doctorat de G.S.G., intitulée La enseñanza del francés en España. Comentario a una bibliografía establecida hasta 1850, dont André Reboullet a depuis longtemps chanté les excellences, a été soutenue il y aplus de quarante ans -en 1956- à l'Université Complutense de Madrid, mais elle est malheureusement restée inédite. L'auteur nous apprend dans sa préface que le sujet lui a été suggéré par le poète et philologue Dámaso Alonso:

Mi antiguo amigo, el ilustre Catedrático de la Facultad de Filosofía y Letras de la Universidad Central, Doctor D. Dámaso Alonso, me sugirió un día este tema en su casa de Chamartín. Nadie lo había tratado en su conjunto y puede decirse que el terreno permanecía en su mayor parte inexplorado. A un profesor consagrado ya hace mucho a la cátedra de Francés, autor además de algunos libros para la enseñanza de este idioma, tenía por fuerza que atraerle la empresa. Puse, pues, manos a la obra y aquí está el fruto de mi modesto trabajo. [Mon ancien ami, l'illustre professeur de la Faculté de Philosophie et Lettres de l'Université Centrale, M. Dámaso Alonso, me suggéra un jour ce sujet dans sa maison du quartier de Chamartín. Personne jusqu'alors ne l'avait traité dans son ensemble et on peut dire qu'il s'agissait d'un terrain pour la plupart inexploré. Un professeur consacré depuis longtemps à sa chaire de français, auteur, en outre, de quelques livres pour l'enseignement de cette langue, ne pouvait qu'être attiré par une telle entreprise, le me mis, donc à l'œuvre et voici le fruit de mon modeste travail] (G. Suárez, 1956: 1). 
Ce prologue de G.S.G. est intéressant pour nous dans la mesure où il confirme l'inexistence alors presque totale, de travaux concernant l'histoire du FLE en Espagne. Il est vrai qu'à cette date, l'extraordinaire Histoire de la Langue française des origines à nos jours de Ferdinand Brunot (1966) était, depuis déjà longtemps, un point de repère indispensable pour qui voulait aborder l'histoire du français non seulement dans les territoires de langue française proprement dits, mais aussi au-delà de leurs frontières:

L'histoire du français langue étrangère ou seconde commence avec Ferdinand Brunot et, tout au long du demi-siècle qui suit la mort de l'historien (en 1938), en France et pour l'essentiel, elle se limite à vulgariser les acquis de l'Histoire de la langue française. C'est-à-dire que Ferdinand Brunot peut être considéré à bon droit comme "le" père fondateur de la SIHFLES et que si cette Société, un jour prochain, publie un Guide $d u$ Chercheur, il s'ouvrira par ce premier conseil: "Lisez Brunot, relisez-le sans cesse" (Reboullet, dans Reboullet et ai, 1995: 148).

7 Mais il faut s'empresser d'ajouter, suivant encore Reboullet (ibid.) que si Brunot est «incontournable, il n'est plus indépassable». Son travail présente certaines lacunes et inexactitudes qu'on ne peut ignorer et qui sont le résultat de la méthode de travail du linguiste. Connaissant bien l'Europe du Nord et Centrale, en particulier l'Allemagne, dont il utilise des sources premières, Brunot s'intéressera beaucoup moins à l'Europe du Sud qu'il connaît d'ailleurs surtout par des témoignages indirects (Chevalier, dans Reboullet et al, 1995: 169-171) et que Reboullet met à juste titre en évidence:

Un déséquilibre [...] se retrouve dans les annexes bibliographiques de l'Histoire où les références primaires ou secondaires concernant l'Europe du Nord l'emportent largement sur celles propres à l'Europe du Sud [...] Des questions sont posées qui en soulèvent d'autres. Par exemple, que vaut, historiquement, l'affirmation selon laquelle le français se serait moins développé en Italie et en Espagne parce que ces deux pays étaient candidats pour leur langue respective à l'universalité alors que les pays de langue allemande ou anglaise n'affichaient pas telle prétention? Ou encore: le rôle de l'émigration protestante française au Nord, était-il si déterminant qu'il fasse méconnaître le rôle des congrégations catholiques au Sud ou les avantages de la parenté latine pour l'apprentissage du français (Reboullet. dans Reboullet étal: 149).

8 La relative pauvreté de matériaux pour décrire la présence du français en Espagne (Lépinette: 159-163) fut d'ailleurs reconnue par Brunot lui-même («certains documents qui n'étaient pas à la Bibliothèque Nationale [de Paris] [lui] ont manqué» [cité par Lépinette, 1995: 159]) ${ }^{5}$; à cela il faut ajouter certains préjugés idéologiques à l'égard de l'Espagne, en particulier celle du 18' siècle. Nombre de prises de position révèlent parfois un certain parti pris et une connaissance pas toujours très profonde de l'histoire et de la culture de notre pays. En définitive, Brunot n'est parfois pas arrivé à dépasser les lieux communs véhiculés par certaines œuvres littéraires et à se défaire complètement de la vision négative que transmirent, entre autres, des voyageurs étrangers. Il va sans dire que ces remarques ne remettent pas l'HLF en question, cet «étonnant monument de la mémoire française», (Chevalier, 1997: 3385), source unique en son genre et toujours à consulter en premier lieu pour qui s'intéresse à l'histoire du français en général, et du FLE en particulier.

9 La thèse de G.S.G. qui comme toutes les œuvres de ce domaine postérieures àla publication de l'HLF a mis à profit les données qu'avait présentées Brunot s'avère pour les chercheurs du domaine du FLE en Espagne une référence obligatoire. Nous ne cachons pas que certaines des options analytiques retenues peuvent sembler quelque peu dépassées actuellement. Ce texte reste néanmoins fondamental, essentiellement 
dans sa partie bibliographique. Comme l'a signalé Marcel Bataillon (dans Suârez Gômez, 1961: 158), dans sa présentation de l'abrégé de la thèse de G.S.G. paru dans la Revue de littérature comparée (XXXV, 1961), le répertoire bibliographique constituait sans doute «l'armature» de l'étude. C'est précisément cette partie que nous retiendrons pour notre commentaire au long des pages qui suivent. Nous essaierons en même temps de mettre en relief certaines décisions parfois problématiques et même contradictoires qui ont été prises lors de la constitution de ce répertoire (mais qui, disons-le, n'annulent en rien le mérite de la tâche réalisée par le professeur madrilène). Signalons que la liste de textes en relation avec l'enseignement du FLE (qui constitue un tiers de la thèse de G.S.G.) a été modifiée et enrichie pour sa publication dans la Revue de littérature comparée. M. Bataillon y présentait ainsi l'ensemble des trois sections dont elle est composée (qui ont fait parallèlement l'objet de trois chapitres successifs dans cette revue), insistant sur la singularité du travail réalisé: «[...] L'auteur a bien voulu l'enrichir encore pour la publier ici, comprenant que son utilité déborde l'éclaircissement des questions d'histoire de la pédagogie et de la philologie qu'il s'était proposées dans ce travail sous la direction de son maître Dámaso Alonso. Les sections $\mathrm{n}$ et III répondent à une utilité encore plus large que ne l'indique le titre ci-dessus; les livres qui y figurent servirent aussi (et d'abord, sans doute) aux Français désireux d'apprendre l'espagnol. Le volume respectif des usagers espagnols et français est une inconnue dont les variations intéressent déjà le comparatiste. La section I, consacrée aux ouvrages de grammaire française rédigés en espagnol, répondait par contre de façon exclusive à l'objet de $\mathrm{M}$. Gonzalo Suárez, objet qui méritait d'autant plus de donner son titre à l'ensemble, que le rayonnement intellectuel et littéraire de la France en Espagne pendant les trois siècles envisagés (1520-1850) a donné lieu à moins d'études précises que le phénomène réciproque» (Bataillon, dans Suárez Gómez, 1961: 158).

10 La première section incluait les ouvrages destinés à l'enseignement, proprement dit, du français aux Espagnols. Il s'agit d'une série assez hétérogène d'ouvrages (méthodes, manuels, grammaires) que l'auteur désigne du nom, pas toujours précis, de grammaires. La deuxième section, intitulée Vocabulaires et dictionnaires, comprend, comme le signale à juste titre Marcel Bataillon, des œuvres dont les destinataires n'étaient pas toujours les Espagnols eux-mêmes, mais parfois des Français désireux d'apprendre la langue espagnole. Elle inclut, d'ailleurs, une bibliographie assez disparate, relevant surtout du genre lexicographique. La troisième section, Recueils de proverbes, récits, romans et autres ouvrages bilingues, plus réduite se révèle aussi plus intéressante. Originairement dans la thèse, elle recevait en espagnol le titre proche (légèrement plus succinct) de Refraneros, relatos y novelas bilingües.

11 Nous voudrions ici considérer les matériaux de la première section, en un choix qui ne signifie pas que les autres sections ne soient pas pertinentes dans l'optique de l'histoire du FLE en Espagne. Nous nous interrogerons sur certaines des options prises par G.S.G., ainsi que sur quelques présences et absences dans cette partie de son répertoire. Notre approche, comparative, consistera à rapprocher les contenus bibliographiques de la thèse soutenue en 1956 et l'inventaire qui figure dans la Revue de littérature comparée cinq ans plus tard et dont les données varient sous certains aspects par rapport à un premier état de la recherche (la thèse). Il convient tout d'abord de faire une observation concernant les limites chronologiques que s'est marqué G.S.G. 
Comme l'a encore signalé M. Bataillon (dans Suárez Gómez, 1961: 158) «l'auteur remonte aussi haut qu'il peut à la recherche des livres qui ont pu servir à procurer quelques notions de français à des Espagnols» et c'est ainsi qu'il atteint le premier quart du $16^{\mathrm{e}}$ siècle. Cependant, ce n'est ni face à une grammaire ni à un manuel spécifiquement destiné à l'enseignement du FLE aux Espagnols que l'on va se trouver dans ce parcours à rebours, mais à un Vocabulario "pour apprendre le français, l'espagnol et le flamand» publié à Anvers en 1520, qui ne nous est pas parvenu et que notre auteur ne connaît que par des références ${ }^{6}$. Il faudra attendre plus d'un demisiècle pour trouver ce qu'on peut considérer comme la première "grammaire» pour l'enseignement du FLE destinée exclusivement aux Espagnols, la Gramática Française de Baltasar de Sotomayor (Alcalá de Henares. 1565). On sait qu'entre-temps, en 1558, le célèbre manuel de Gabriel Meurier, vrai best-seller de son époque, avait vu le jour à Anvers, mais ce dernier, relevant du genre multilingue, fréquent au $16^{\mathrm{e}}$ siècle, intéresse donc aussi n'importe lequel des groupes nationaux concernés désireux d'apprendre l'une des langues incluses ${ }^{7}$. Ainsi, la limite chronologique inférieure du parcours de G.S.G. n'est ni 1565 ni 1558, mais celle du Vocabulario d'Anvers. Cependant, l'année 1850, qui représente le terme supérieur dans la recherche, qualifiée d'arbitraire par $\mathrm{M}$. Bataillon nous semble pourtant plus pertinent. En effet, à partir de la seconde moitié du $19^{\mathrm{e}}$ siècle, on constate l'existence, en grand nombre, «de livres pour l'étude du français par les Espagnols (et réciproquement)» (Bataillon, dans Suárez Gómez, 1961: 159), fait qui complique à l'extrême la tâche du chercheur - et de là sans doute l'intérêt de G.S.G. pour mettre un terme à l'enquête. Cette multiplication de manuels pour le FLE est un effet de la Loi Moyano (1857), qui conduisit à l'institutionnalisation des langues étrangères, notamment du français, intégré dans l'enseignement secondaire à partir de cette date. Face à cette surabondance, G.S.G. a opté pour arrêter son enquête dont l'objectif se trouve, de ce fait même, limité à une époque où l'enseignement de la langue française n'était pas encore institutionnalisé en Espagne (précisons pourtant que cet enseignement avait été intégré dans les Planes de estudios de certains établissement religieux et militaires dès le $18^{\mathrm{e}}$ siècle) et les «livres de français» étaient, pour cette raison, en nombre relativement réduit ${ }^{8}$.

En ce qui concerne les contenus du répertoire élaboré, aussi bien dans la thèse de 1956 que dans l'article de la Revue de littérature comparée (1961), il faut souligner d'abord leur extension. Dans la partie Grammaires, qui a fait, sans aucun doute, tout particulièrement l'objet de l'intérêt du professeur madrilène, G.S.G. inclut quatre-vingt-un titres, dont les dates d'édition comme nous l'avons signalé plus haut, vont de 1558 à $1850^{\circ}$. Le répertoire de 1961 (Revue de littérature comparée; à partir de maintenant: RLC) offre dix titres de plus que la thèse de doctorat (à partir de maintenant: TD), en plus de modifications et de données complémentaires. La comparaison entre TD et RLC permet de faire d'abord remarquer que RLC a exclu un livre de 1660 qui figurait dans TD. La raison de cette exclusion est que, malgré son titre, Nouvelle grammaire espagnole et française (Paris, L. Chanhoudry), l'ouvrage est une grammaire exclusivement espagnole publiée en France, et donc non destinée à des usagers désireux d'apprendre le français. Cette disparition est compensée quelques lignes plus bas, par l'apparition dans cette même liste, d'une méthode d'apprentissage multilingue concernant non seulement le français et l'espagnol mais aussi d'autres langues (entre autres, le polonais) ${ }^{10}$. En fait, il s'agit de l'un de ces manuels qui avaient été en vogue cent cinquante ans auparavant. Il n'est pas facile de comprendre pourquoi G.S.G. a intégré cette œuvre dans RLC (aucune référence n'y était faite dans TD). De même, un autre ouvrage qui ne diffère que peu du 
précédent, le Thésaurus fundamentalis quinque linguarum (Latina, Hispánica, Gallica, Itálica et Germánica) de Juan Ángel de Sumarán -sans doute consulté à la Bibliothèque Nationale de Madrid- est cité par Suárez Gómez aussi bien dans TD que dans RLC. Cette œuvre, publiée en $1626^{11}$ cinq ans avant la première édition du Janua Linguarum reservata de Comenius (Leszno, Pologne, 1631), participe, dans une certaine mesure, du même état d'esprit que celui que manifeste l'ouvrage du pédagogue tchèque, bien qu'on ne puisse parler d'une influence directe du premier sur le second. Il ne faut pourtant pas oublier que Sumarán était professeur de langues à l'université bavaroise d'Ingolstadt et devait donc être en contact avec l'élite intellectuelle d'Europe centrale. Cet aspect rend intéressante la mention dans le répertoire de cet auteur pratiquement ignoré aussi bien des historiens de FLE que de ceux de l'espagnol langue étrangère ${ }^{12}$. La biographie nous renseigne aussi sur le parcours européen de Sumarán qui «voyagea à travers l'Europe, combattit en Flandre, enseigna des langues, en particulier à de grands personnages, et fut professeur de langues étrangères à l'université d'Ingolstadt, en Bavière». Elle nous permet de connaître une caractéristique du pédagogue espagnol: son enthousiasme pour l'enseignement des langues étrangères qui est défendu en termes éloquents dans la dédicace à Don Francisco de Moneada ouvrant le Thésaurus. Dans le même esprit, Fray Diego de la Encamación (1624) avait aussi remarqué le manque d'intérêt des Espagnols pour l'étude des langues vivantes ${ }^{13}$ :

He compuesto este dificultosa obra sobre todo para que otros se sirvan con facilidad de lo que yo he alcançado con grandissimo trabajo: Principalmente nuestra nación Española, poco curiosa de saber lenguas extrangeras, cosa muy indigna de una nación tan nombrada en estos siglos; teniendo a tantas naciones debaxo de su dominio, cuyas lenguas devrían saber perfectamente por muchos respetos. Porque por las lenguas se viene a saber en tierras estran-geras en poco tiempo lo que nunca sin ellas se supiera ni entendiera en mucho; por las lenguas se mantiene la buena correspondencia y amistad. Por las lenguas se oye benignamente las relaciones, quexas, y diferencias que en la República ocorren. y a distinguir lo bueno de lo malo sin haver menester intérprete, porque muchas veces se desea de comunicar a un Principe o Señor, por no querella comunicar a ninguno sino a él mesmo, ni que venga por terceros, sino por su mesma boca, o escritura sabiendo que la entiende ... [J'ai composé cet ouvrage plein de difficultés pour que d'autres que moi se servent avec facilité de ce que j'ai atteint avec un très grand effort: en particulier notre nation espagnole, peu curieuse de savoir des langues étrangères, une chose très indigne d'une nation si renommée dans ces derniers siècles; qui a tellement de nations sous son empire, dont les langues devraient être connues pour de nombreuses raisons. Car grâce aux langues on arrive à savoir dans des terres étrangères, en peu de temps, ce que sans elles on mettrait trop longtemps à savoir et à comprendre. Grâce aux langues on entretient une bonne correspondance et amitié. Grâce aux langues on écoute volontiers les rapports, les plaintes et les différences qui ont lieu dans la république, en discernant le bon d'avec le mauvais sans avoir besoin d'interprète, car on souhaite souvent communiquer quelque chose à un prince ou à un seigneur, sans vouloir la communiquer à aucun autre qu'à lui-même, et sans que cela provienne d'une tierce personne, mais de sa bouche même, ou de son écriture en sachant qu'il la comprend ...] (Suârez Gômez, 1956: 94-95).

G.S.G. nous présente, aussi bien dans TD que dans RLC, un corpus de manuels et de grammaires pour l'apprentissage du FLE par les Espagnols, publiés tant en Espagne qu'à l'étranger du $16^{\mathrm{e}}$ au $18^{\mathrm{e}}$ siècles, spécialement dans la première moitié de ce dernier, qui est caractérisé par son ampleur. La plupart des titres maniés par les chercheurs actuels figuraient déjà dans la liste de notre auteur. U suffit de comparer celle-ci avec les répertoires récents, en particulier Supiot (1996: 313-328) -sans aucun doute le plus 
complet concernant l'époque 1648-1815- pour constater le fait. D'ailleurs, cet auteur reconnaît sa dette envers G.S.G. et il cite (1996: 312) TD comme «référence à laquelle il faut obligatoirement se rapporter», tout en signalant (ibid.)la nécessité d'une mise à jour devant conduire à «l'établissement d'une bibliographie qui tiendrait compte des différentes éditions ainsi que des variantes, contrefaçons, etc.». Supiot souligne aussi le point «le plus faible» des travaux de G.S.G. qui n'a tenu compte que de certaines éditions des ouvrages cités, consultés dans les bibliothèques (presque exclusivement la B.N. de Madrid et celle de Paris). Cette limitation a, entre autres conséquences, que la grammaire de Pierre-Paul Billet figure dans sa $2^{\mathrm{e}}$ édition (Madrid, Bemardo de Villadiego, 1688), sans qu'il y ait de référence à celle d'Anvers (H. et C. Verdussen, 1687). Les deux éditions qui figuraient déjà dans Stengel (1689) -qui n'était pas certain cependant de l'armée qu'il indiquait dans son catalogue-, seront normalement reprises par Supiot (1996) et Fischer (1996).

Soulignons que, comme nous l'avons déjà mentionné, l'extension des listes de G.S.G. provient, en partie, de l'intégration dans son répertoire de n'importe quel ouvrage destiné à des apprenants espagnols, indépendamment du lieu de publication (Supiot, 1996, dont le critère, plus restrictif, a été de se limiter aux textes de FLE pour Espagnols édités en Espagne, est donc moins étendu, bibliographiquement parlant). II figure, par exemple, dans G.S.G. (TD et RLQun ouvrage peu cité, la Grammaire nouvelle françoise et espagnole en douze leçons dialogismes, de José Dacosta fils ${ }^{14}$, publiée chez un imprimeur de Londres en 1752, dont la notice nous apprend que:

Este "maestro de lenguas" pertenecía a una familia judía de origen español, Su padre se había dado a conocer como autor de un Tratado de Cortesía y Política (Amberes, 1726). Las doces lecciones dialogadas de Dacosta exponen concisa y claramente en forma bilingüe la pronunciación y la morfología. En las últimas páginas del libro hay algunas reglas de sintaxis y varias observaciones ortográficas. Con ésta y la Contaut diríase que se produce una saturación de gramáticas francesas que requiere una pausa de cerca de cuarenta años, interrumpida sólo, pero espléndidamente, por el Arte de Chantreau [...].

[Ce "maître de langues" appartenait à une famille juive d'origine espagnole. Son père s'était fait connaître comme auteur d'un Traité de Civilité et Politique (Anvers, 1726). Les douze leçons dialoguées de Dacosta exposent d'une façon concise et claire sous forme bilingue la prononciation et la morphologie. Dans les dernières pages du livre il y a quelques règles de syntaxe et certaines observations sur l'orthographe. On pourrait dire qu'avec cette grammaire et celle de Contaut une surabondance de grammaires françaises se produit, ce qui contraint à une pause de près de quarante ans, interrompue seulement, mais d'une manière splendide, par l'Art de Chantreau] (c'est nous-mêmes qui traduisons directement de l'original de Suárez Gómez) (1956: 117).

Plusieurs pages de TD (1956: 105-107) sont parallèlement consacrées à l'abbé Vayrac, auteur d'une vaste grammaire éditée en deux gros volumes (453 pages le premier et 964 pages le second, Paris, 1714). G.S.G. décrit aussi brièvement la vie de l'abbé et ses caractéristiques: historien érudit, il avait passé de longues années en Espagne avant de rentrer à Paris ${ }^{15}$. C'est peu après son retour en France qu'il aurait publié son livre, à une date d'ailleurs spécialement significative, l'année où la paix qui mit fin à la Guerre de Succession d'Espagne rapprocha les deux pays et resserra leurs liens d'amitié. Le responsable de la censure y illustre l'état d'esprit né de la nouvelle situation politique:

[...] juzgo que los Españoles que se dedican al estudio del Idioma Francés, podrán sacar de este libro grandissimas ventajas, y que de él resultará el estrechar el vínculo de la unión que ay entre las Naciones Española y Francesa, y facilitará una 
buena correspondencia en el Comercio con la comunicación de las dos lenguas. [Je pense que les Espagnols qui se consacrent à l'étude de la langue française pourront tirer de ce livre de nombreux avantages, et qu'il servira à resserrer les liens de l'union qui existe entre les nations espagnole et française, et cela facilitera de bonnes relations dans le commerce moyennant la communication entre les deux langues.]

17 La grammaire française de l'abbé Vayrac est décrite par G.S.G., comme une «vaste et excellente grammaire qui, outre les études nécessaires de phonétique et morphologie, contient une syntaxe très précise inspirée principalement des règles de Régnier Desmarais et du père Buffier». L'ouvrage mentionne aussi les tentatives successives de réforme de l'orthographe française et inclut un «Traité de la Poésie au profit de tous ceux qui voudront se consacrer à connaître les vers et à les composer; suivi des normes de la conversation (titres, formules de politesse, etc.) employées en France, puis finalement un formulaire pour écrire des lettres». Rappelons que le fait était courant à l'époque: Billet (1673) fermera aussi son ouvrage par un traité de poésie. En Espagne, un auteur de grammaire française de la première moitié du 18' siècle, De la Torre y Ocón (1728), citera aussi Regnier-Desmarais et Buffier comme source, et Núñez de Prado (1728) s'est également inspiré de ce dernier, jésuite comme lui ${ }^{16}$.

18 Signalons que Dacosta et Vayrac figurent dans Stengel [1889] (Billet également, nous l'avons vu, aussi bien dans les premières éditions de Madrid que celle d'Anvers). Nul doute que l'une des sources de G.S.G. a été le répertoire de l'érudit allemand.

L'Arte de Hablar bien el Francés de Pierre-Nicolas Chantreau est intégré dans un chapitre qui le concerne seul. G.S.G. a eu le mérite d'être l'un des premiers ${ }^{17}$ à souligner la valeur linguistique de l'ouvrage, au-delà de l'intérêt historique de la biographie de cet auteur, aussi prise en compte dans TD.

En ce qui concerne les exclusions de G.S.G. \{exclusions en fonction de ses propres critères, évidemment), mentionnons, par exemple, les Reglas gramaticales para aprender la lengua española y francesa [...] publiées à Oxford en 1586, qui sont présentées aussi bien dans TD que dans $R L C$, sans nom d'auteur, alors qu'il n'y a plus de doute aujourd'hui qu'elles furent écrites par Antonio del Corro (voir Ramajo Caño, 1987), Espagnol qui embrassa la religion réformée et, pour cette raison, dut se réfugier en France puis en Angleterre où il enseigna l'espagnol (il exerça même les fonctions de pasteur au sein de la communauté espagnole de Londres). G.S.G. se limite à signaler dans TD que cet ouvrage avait été publié chez Joseph Barnes à Oxford. Il ajoute dans RLC qu'il s'agit d'un ouvrage cité par Fouché-Delbosc dans sa Bibliographie Hispano-Française (1912), sans aucune autre référence (il ne fait pas non plus allusion à un second ouvrage d'Antonio del Corro probablement la traduction anglaise de la version de 1586, quelque peu remaniée et augmentée- publié quatre ans plus tard, en 1590: The Spanish Grammer. With certaine rules teaching both the Spanish and french Tongues).

21 Le $18^{\mathrm{e}}$ siècle, qui nous intéresse tout spécialement, montre, si nous comparons G.S.G. avec A. Supiot et B. Lépinette (dans García-Bascuñana et al, 1996) un certain nombre de lacunes. Des ouvrages sans doute -au moins pour certains-déjà catalogués dans des bibliothèques espagnoles dans les années 50 sont ignorés par G.S.G. aussi bien dans TD que dans RLC.

C'est le cas de neuf ouvrages de la seconde moitié du 18 e siècle (Supiot, 1996: 320-323): Roca y Maria (1750) ${ }^{18} \backslash$ Rousseau (1754) ${ }^{19}$, Sarrio (1768) ${ }^{20}$, Magín Talles (1773) ${ }^{21}$, González Cañaveras (1781) $)^{22}$, Laborda (1784) ${ }^{23}$ Martínez Saavedra ${ }^{24}$ (1791), Jo-vellanos (1794) ${ }^{25}$, 
Boyer $(1799)^{26}$. La seconde moitié du $18^{\mathrm{e}}$ siècle serait, paradoxalement, si on tient compte de l'importance de cette époque dans l'histoire du FLE, la partie la moins complète du répertoire de G.S.G., ce qui nous fait penser que ce dernier n'a pas cru devoir (ou n'a pas pu) faire de recherche hors des B.N. déjà nommées (Madrid et Paris). Ce n'est donc pas par hasard que cinq de ces neuf manuels répertoriés par Supiot (Roca y Maria, Rousseau, Sarrio, González Cañaveras et Boyer ${ }^{27}$ se trouvent dans des bibliothèques de province.

On devra admettre (au moins provisoirement, i.e.en fonction de l'état de nos connaissances actuelles) que le meilleur du répertoire de G.S.G. concerne le $16^{\mathrm{e}}$ et le $17^{\mathrm{e}}$ siècles, en sa partie consacrée aux grammaires et manuels proprement dits (ainsi que dans celle qui répertorie dictionnaires et recueils de textes). Cet apport nous paraît d'autant plus précieux qu'il a recensé une période assez peu connue, au moins au moment où la thèse de notre auteur a été soutenue. En revanche, au fur et à mesure que nous nous rapprochons du terme chronologique du recensement, et que le nombre des ouvrages pour l'enseignement $d u$ français croît, l'on constate chez G.S.G. une plus grande difficulté pour repérer les matériaux et les sélectionner de façon pertinente. Il faut ajouter que G.S.G. a ignoré dans son recensement les critères pédagogiques -il ne différencie pas les grammaires en fonction de leurs destinataires, en Espagne ou hors de ce pays- et qu'il n'a pas semblé non plus extrêmement intéressé par les contenus grammaticaux sur lesquels il ne s'étend qu'exceptionnellement. Son étude reste externe, typique d'un auteur de répertoire bibliographique, se limitant à situer les œuvres et les auteurs dans leur contexte purement historique.

Malgré les limitations que nous venons de souligner, les résultats des recherches effectuées par G.S.G. constituent indéniablement le point de départ de l'histoire du FLE en Espagne. L'œuvre du professeur madrilène y tient la première place, non seulement par sa situation chronologique (les années 50), mais aussi par l'importance du recensement qu'elle inclut. En même temps, elle permet de faire le point sur l'histoire du FLE hic et nunc, de mieux prendre conscience de ce qui est déjà fait, mesurant, avec un recul suffisant, ce qui reste à faire. André Reboullet (1987: 55-60) l'a dit (son observation est tout spécialement applicable à notre champ espagnol): «la recherche sur l'enseignement du français langue étrangère, n'est plus ce qu'elle était: un véhicule sans rétroviseur qui brûle les étapes. [...] C'est l'heure des bilans et des rétrospectives». Bref, nous sommes déjà dans l'histoire de l'histoire du FLE.

\section{BIBLIOGRAPHIE}

BATAILLON, Marcel (1961): «Présentation» dans G. Suárez Gómez «Avec quels livres les Espagnols apprenaient le français (1520-1850)», dans Revue de Littérature comparée, t. XXXV, pp. 158-160 (158-171).

BRUNOT, Ferdinand (1966-lère éd. 1905): Histoire de la langue française des origines à nos jours, Paris, Armand Colin. 
CHEVALIER, Jean-Claude (1995): «F. Brunot, l'Histoire de la Langue française et le français à l'étranger. Conditions d'enquête et méthodes d'investigation», dans A. Reboullet et al, «Ferdinand Brunot et l'Europe du Sud», Documents pour l'histoire du français langue étrangère ou seconde, $\mathrm{n} .^{\circ} 16, \mathrm{pp}$.

167-171.

CHEVALIER, Jean-Claude (1997): «L'histoire de la langue française» de Ferdinand Brunot, dans Nora, P. (dir.), Les Lieux de mémoire, Paris, Gallimard «Quarto», pp. 3385-3419, vol. 3.

FERNÁNDEZ Fraile, María Eugenia (1995): La enseñanza/aprendizaje del francés como lengua extranjera en España (1767-1936: objetivos, contenidos y procedimientos), Granada, Universidad de Granada. FISCHER, Denise (1996): «La Gramática Francesa de Pierre Paul Billet: contenus et méthode», dans García-Bascuñana, L; Lépinette, B. et Roig, C.(éds.), L'«universalité» du français et sa présence dans la Péninsule Ibérique, Documents pour l'histoire du français langue étrangère ou seconde, $\mathrm{n} .^{\circ} 18, \mathrm{pp}$. 212-223.

FOUCHÉ-DELBOSC, Raymond (1912-1914): Bibliographie hispano-française, 3 vols., New York, H.S.U.A. GARCÍA-BASCUÑANA, Juan; Lépinette, Brigitte et Roig, Carmen (1996): $L^{\prime}$ «universalité» du français et sa présence dans la Péninsule Ibérique, dans Documents pour l'histoire du français langue étrangère ou seconde, $n .^{\circ} 18$.

LÉPINETTE, Brigitte (1995a): «F. Brunot et l'Espagne», dans A. Reboullet et al, «Ferdinand Brunot et l'Europe du Sud», Documents pour l'histoire du français langue étrangère ou seconde, n. ${ }^{\circ} 16, \mathrm{pp}$. 159-166.

LÉPINETTE, Brigitte (1995b): «La grammaire contrastive franco-espagnole de la première moitié du $18^{\mathrm{e}}$ siècle. Analyse de six ouvrages édités en Espagne», Historiographia Lingüistica, vol XXII, 1/2, pp. 27-74.

LÉPINETTE, Brigitte (1995c): «El arte de hablar bien francés (1781) de P.-N. Chantreau (1741-1808), grammaire pour l'enseignement du français aux Espagnols», Le français moderne, $\mathrm{n} .{ }^{\circ} 2, \mathrm{pp}$. 138-165.

RAMAJO CAÑO, Antonio (1987): Las gramáticas de la lengua castellana desde Nebrija a Correas, Salamanca, Universidad de Salamanca.

REBOULLET, André (1987): «Pour une histoire de l'ensiegnement du F.L.E.», Le français dans le monde, n. ${ }^{\circ} 208$, pp. 56-60.

REBOULLET, André (1995): «Présentation» dans A. Reboullet et al. «Ferdinand Brunot et l'Europe du Sud», Documents pour l'histoire du français langue étrangère ou seconde, n. ${ }^{\circ}$ 16, pp. 148-149.

ROLDAN PÉREZ, Antonio (1976): «Motivos para el estudio del español en las gramáticas del siglo XVI», Revista de Filología Española, LVTJI, pp. 201-229.

SÁNCHEZ PÉREZ, Aquilino (1992): Historia de la enseñanza del español como lengua extranjera, Madrid, SGEL.

STENGEL, Edmund (1976²); Chronologisches Verzeichnis französischer Grammatiken, Neu Herausgegeben mit einen Anhang von H.-J. Niederehe, Ams-terdam/Philadepphia, John Benjamins.

SUÁREZ GÓMEZ, Gonzalo (1956): La enseñanza del francés en España (Comentario a una bibliografía establecida hasta 1850), thèse de doctorat inédite, Université Complutense de Madrid. SUÁREZ GÓMEZ, Gonzalo (1961): «Avec quels livres les Espagnols apprenaient le français (1520-1850)», Revue de Littérature comparée, t. XXXV, pp. 158-171; 330-346; 512-523. 
SUPIOT, Alberto (1996): «Les manuels de Français Langue Étrangère en Espagne entre 1648 et 1815. Approche bibliographique», dans J. García-Bascuñana, B. Lépinette et C. Roig (éds.), L'universalité du français et sa présence dans la Péninsule Ibérique, Documents pour l'histoire du français langue étrangère ou seconde, $\mathrm{n} .^{\circ} 18, \mathrm{pp} .313-328$

\section{NOTES}

1. Il faut citer, en particulier, le vaste ensemble: Christ,H. \& Rang, H.-J. (1985): Fremdsprachenunterricht unter staatlicher Verwaltung 1700-1945, 7 tomes, Tübingen, Gunter Narr Verlag.

2. Stockholm, Akademilitteratur, 1980.

3. Minerva, N. (1996): Manuels, maîtres, méthodes. Repères pour l'histoire de l'enseignement du français en Italie, Bologne, CLUEB; Minerva, N. et Pellandra, C. (1997): Insegnare il francese in Italia. Repertorio anatitteo di manuali pubblicati dal 1625 al 1860, Bologne. CLUEB

4. Brigitte Lépinette prépare un article intitulé «Vhistoire d'une (courte) histoire. Le FLE en Espagne. Bilan (1956-1998). Perspectives». La publication est prévue dans Documents pour l'histoire du français langue étrangère ou seconde; $\mathrm{n}^{\circ} 21$.

5. «Ce manque de matériel espagnol pour le sujet qu'il a traité constitue sans aucun doute une des causes du caractère parfois rapide de certaines analyses (en particulier celles du chapitre IV)».

6. Suarez Gômez signale que ce livre avait été cité par Fouché-Delbosc: Bibliographie HispanoFrançaise, 1912, p. 20

7. Le livre de G. Meurier, publié à Anvers, chez J. Van Waesberghe, a pour titre Conjugaisons, règles et instructions mout propres et nécessairement requises pour ceux qui désirent apprendre François, Italien, Espagnol et Flamen dont la pluspart est mise par manière d'interrogations et Responses. Il était suivi d'une Brève instruction contenante la manière de bien prononcer le François, Italien, Espagnol et Flamen (les deux ouvrages étaient reliés ensemble).

8. La thèse de doctorat de María Eugenia Fernández Fraile (1995) recouvre en partie la fin de la période traitée par Suárez Gómez et en complète même certains des aspects pour le dernier tiers du $18^{\mathrm{e}}$ siècle et la première moitié du $19^{\mathrm{e}}$.

9. Le $n^{\circ} 81$ n'a pas de date exacte, l'auteur n'a signalé que les deux premiers chiffres [18...].

10. Cet ouvrage, publié en 1701, dont le titre complet est Méthode courte et facile pour apprendre en peu de temps le latin, le français, l'italien, l'espagnol, l'allemand, le flamand, l'anglois et le polonais, se trouverait à la Bibliothèque Mazarine.

11. G.S.G. a corrigé dans RLC la date qu'il proposait dans TD. L'année 1649 (TD) pour l'ouvrage de Sumarán était erronée et il la corrigea. D'après G.S.G., Sumarán avait dédie son livre à Don Francisco de Moneada, comte d'Osona, ambassadeur d'Espagne auprès de l'Empereur. Pour cette raison, la date de publication ne pouvait être 1649: Don Francisco de Moneada, ancien vice-roi des Flandres et auteur d'un ouvrage historique sur l'expédition en Grèce et en Orient des Catalans et Aragonais ( 1623), était mort en 1635.

12. Aquilino Sánchez Pérez dans son Historia de la enseñanza del español como lengua extranjera ne fait aucune allusion à l'ouvrage de Sumarán.

13. Ce désintérêt des Espagnols du $16^{\mathrm{e}}$ et $\mathrm{du} 17^{\mathrm{e}}$ siècles pour les langues autres que la leur est expliqué dans Roldan (1976).

14. Cette mention -Dacosta fils- provient sans doute du fait que le fils voulait profiter de la notoriété du père dans certains milieux de l'exil judéo-espagnol.

15. A. Sânchez Pérez (1992: 165-166) qui se base certainement sur G.S.G. affirme que Vayrac avait séjourné à la Cour d'Espagne durant près de vingt-cinq ans. Cette longue période lui avait permis 
d'acquérir une grande maîtrise de la langue espagnole et de publier aussi une grammaire de cette langue à l'usage des Français (1708), dont la seconde édition, la plus connue, est de 1714, c'est-àdire l'année où fut publiée sa grammaire française. Cette seconde édition, «revue, corrigée et augmentée de plus des deux tiers», parut aussi à Paris chez Pierre Witte, l'imprimeur de son Arte Froncés.

16. Lépinetel, $1995 \mathrm{~b}$.

17. Il faudra attendre la dièse de Nuria Moreu Huet [Pierre Nicolas Chantreau (1741-1808) et sa grammaire, Barcelona, Publicacions de la Universität de Barcelona, 1990] pour compléter exhaustivement nos connaissances sur la tâche linguistique de ce «maître de langue française». Voir aussi Lépinette, $1995 \mathrm{c}$.

18. Arte francés o Nuevo método facilissimo para leer, hablar y escrivir francés, conforme a lo más moderno. Barcelona, Francisco Suriá.

19. Rudimentos de la Lengua Francesa, à Extracto de Preceptos breves, y claros para su prompta inteligencia, Vaíladolid, Alonso del Riego.

20. Rudimentos de la Lengua Francesa [..] para instrucción de la Nobleza Española del Colegio Andresano Valencia, Benito Monfort.

21. Rudimentos de la Pronunciación Francesa para el uso de los Caballeros del Real Seminario de Nobles Madrid, Antonio Mayoral

22. Cartilla francesa o combinaciones generales para la lectura .... Cádiz, Luis de Luque y Leyva.

23. Reglas para la Pronunciación de la Lengua Francesa, Madrid, Antonio de Sancha.

24. Compendio de la Gramática Francesa, explicada por partes, y aumentada de las reglas generales de la Orthografía..., Sevilla, Vázquez e Hidalgo.

25. Rudimentos de la gramática francesa. Cette grammaire faisait partie d'un Cours d'Humanités, conjointement avec une grammaire anglaise et une grammaire espagnole rédigées par Jovellanos luimême (incluse actuellement dans Cándido Nocedal éd., Obras de Jovellanos. Madrid, Atlas «BAE», 1963).

26. Método facilísimo para leer y hablar francés sin socorro de maestro, Barcelona, Antonio Sastres.

27. Les bibliothèques dans lesquelles se trouvent des exemplaires des cinq ouvrages nommés sont les suivantes (Supiot, 1996); Roca y Maria (Biblioteca Universitaria de Barcelone); Rousseau (Biblioteca Sta Cruz de Valladolid et Biblioteca Universitaria de Saint-Jacques de Compostelle); Sarrio (Biblioteca Municipal de Valence); Gonzalez Cañaveras (Biblioteca del Palacio Real de Madrid); Boyer (Biblioteca de Catalunya de Barcelone).

\section{AUTEURS}

\section{JUAN FRANCISCO GARCÍA BASCUÑANA}

Université Rovira i Virgili (Tarragone)

\section{BRIGITTE LÉPINETTE}

Université de Valence

\section{CARMEN ROIG}

Université de Cantabria 